\title{
DEVELOPMENT AND IMPLEMENTATION OF A SECURE PRODUCTION NETWORK
}

\author{
Isabella Reithner ${ }^{1}$, Maximilian Papa ${ }^{1}$, Bernhard Lueger $^{1}$, Merim Ćato ${ }^{2}$, \\ Siegfried Hollerer ${ }^{3} \&$ Rolf Seemann ${ }^{1}$
}
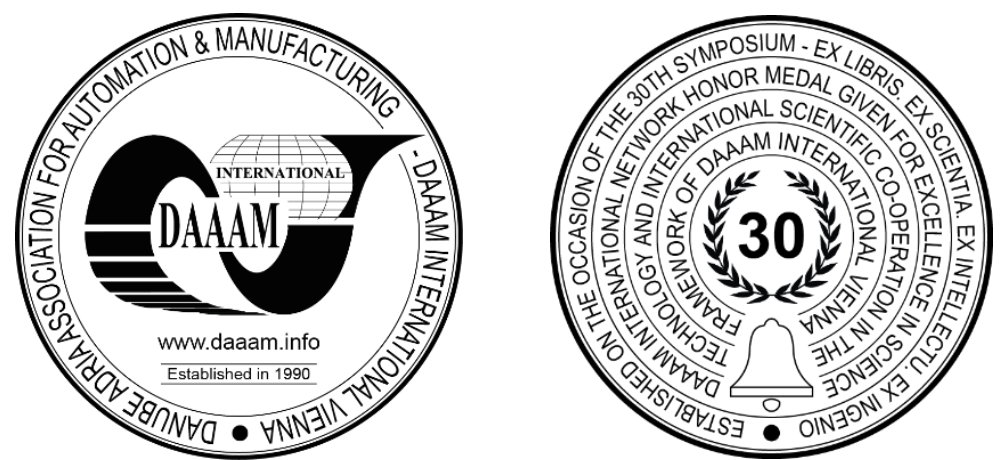

This Publication has to be referred as: Reithner, I[sabella]; Papa, M[aximilian]; Lueger, B[ernhard]; Cato, M[erim]; Hollerer, S[iegfried] \& Seemann, R[olf] (2020). Development and Implementation of a Secure Production Network, Proceedings of the 31st DAAAM International Symposium, pp.0736-0745, B. Katalinic (Ed.), Published by DAAAM International, ISBN 978-3-902734-29-7, ISSN 1726-9679, Vienna, Austria DOI: $10.2507 / 31$ st.daaam.proceedings. 102

\begin{abstract}
With the rise of the intelligent production environments the consistent automatization of ordering, production and shipping is intended. A certain company network is therefore necessary, which is basically segmented in IT and OT. Unfortunately, these two networks are often not properly secured or wrong configured leading to vulnerabilities for cyberattacks and thus to safety hazards due to inseparable interaction. Therefore, a network architecture, segmented into six subnets will be designed in this work. This proposed network of the production plant consists of an OPC UA server and multiple clients (based on the open62541 library), a mobile robot and state-of-the-art security measures. Finally, a penetration test by TÜV TRUST IT TÜV AUSTRIA GmbH team was executed to validate this setup and to discover remaining security vulnerabilities. Subsequently the network-architecture and the security-measures based on the IEC 62443 requirements were improved and as a result to be operational in a secure environment.
\end{abstract}

Keywords: Network segmentation; IT Security; Security vulnerabilities; IEC 62443; OPC UA (open62541 library)

\section{Introduction and current limitations}

The future of production sees its advantages in flexibility and modularity. This so-called fourth industrial revolution leads to the dissolving of the classical automation pyramid, where components of different manufacturers are communicating across all levels of this pyramid using standardized communication protocols. Currently field bus protocols are the common way for exchanging information between industrial control systems (ICS) [1]. The disadvantage of these bus protocols is that they are only applicable on the lower levels of the pyramid. Therefore, standardized protocols based on Ethernet with real time-features are replacing proprietary field bus communication systems [1].

\footnotetext{
${ }^{1}$ University of Applied Sciences (UAS) Technikum Wien (Fachhochschule Technikum Wien)

2 TÜV AUSTRIA HOLDING AG

${ }^{3}$ TÜV TRUST IT TÜV AUSTRIA GmbH
} 
Ethernet based communication protocols are a well-known standard and can be easily implemented on different operating systems as well as on microcontrollers [2]. The further this trend progresses, the less it is possible to draw a line between Information Technology (IT) and Operational Technology (OT). Historically these two systems were physically separated. In the pyramid, the IT systems are located at the top levels, whereas OT systems are located at the lower levels. The differences between an IT and an OT environment are the place of deployment, service life and real-time requirements. OT systems are used in critical infrastructures, manufacturing and transportation where they are supposed to control physical processes and provide data to the enterprise 24/7/365 without interruptions [3].

Industry 4.0 components are able to communicate with any endpoints, even across these levels. Especially with cloud systems, currently one of the strongest trends in Industry 4.0, data is no longer transmitted via cables or wires, but can be virtualized and transferred between sensors and devices. For this reason, the classic pyramid dissolves to a network of cyber-physical systems (Fig. 1) [4], where OT devices are able to communicate directly to the enterprise. The only distinguishing feature of IT and OT is the requirement for real time-features and functional safety (potential to harm people), which is not given in the Manufacturing Execution System (MES) or the Enterprise-Resource-Planning (ERP) systems [4].

To advance the trend of cyber-physical systems a pilot factory (UAS Technikum Wien Digital Factory ${ }^{1}$ ) was build up at the UAS Technikum Wien. Latest Industry 4.0 technologies and scenarios can be tested and implemented there. Besides implemented robot systems, also data models for cloud computing were created and machine learning techniques are used for manufacturing smart products.

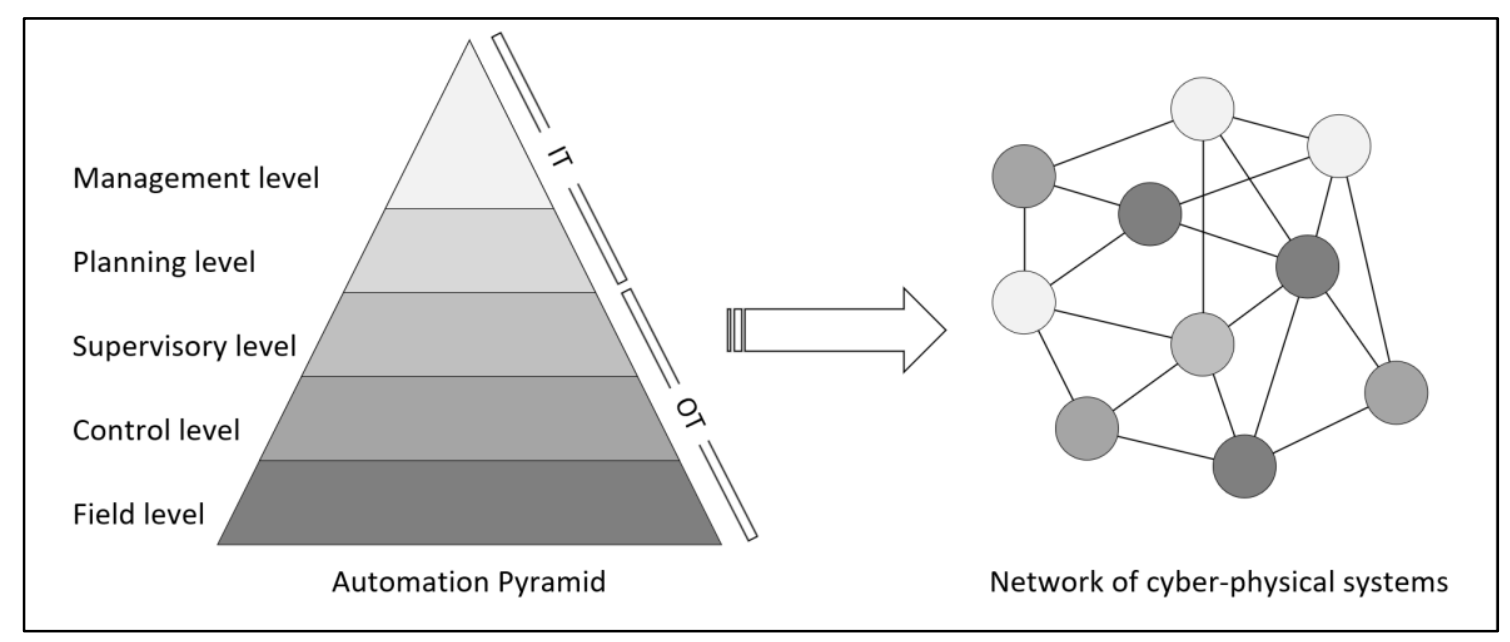

Fig. 1. Transition from the rigid automation pyramid to a flexible network of cyber-physical systems [5]

In order to secure industrial control systems in production, a number of challenges arise from the merge of IT and OT. The interaction of components gets more complex [4] - considering that more data is available - and individual products increased decentralization approaches are pursued. Furthermore, the priorities and requirements are different in IT and OT systems [4]. For example, the priority of IT systems is confidentiality while it is availability for OT systems [6]. The last challenge is an interdisciplinary and device-independent approach of communication [4].

In order to overcome these challenges in a production network, the IT and the OT segment of the network need to be able to communicate with a standardized communication protocol. In addition, security measures need to be implemented, because standardized protocols and web-enabled components linked to ICS or mobile robots, contain the danger of cyberattacks, which can lead to safety hazards due to inseparable interaction. However, in Austrian companies there is still a lack of awareness for this type of attacks [4].

Based on the European Manufacturing Survey 2018 [7], the Federal Ministry for Climate Action, Environment, Energy, Mobility, Innovation and Technology (BMK) Austria presented specific results according to the awareness of Austrian companies about security. Large companies are implementing increasing amounts of Industry 4.0 technologies and security measures, leading to a new security-conscious trend. This trend is very important considering the rising number of cyber-threats. However, the survey also shows that one third of businesses (small, medium, large) cannot assess whether cyber-attacks pose a relevant threat or not. [4]. In addition, each production is different and must therefore be adapted to the specific application and requirements. The structure of the production network cannot be generalized. A method for planning and implementing safety and security in production has already been presented as an assistance for the problem of the individuality of production plants [8].

In order to address the above-mentioned problems and to improve the security awareness of the companies, the stateof-the-art secure industrial communication, will be primary discussed in this paper, followed by the evaluation of the network setup and segmentation in a test factory by TÜV TRUST IT TÜV AUSTRIA GmbH.

$\underline{\underline{1} \text { https://www.technikum-wien.at/digitale-fabrik/ [Accessed: 21.09.2020] }}$ 


\section{Secure industrial communication}

As mentioned above, the combination of sensors, measuring systems and safety devices (as used in machines and mobile robots) typically needs to communicate with a higher-level control system, which makes the manufacturer independent communication necessary. Focusing on the communication between control systems and robots, the uniform OPC UA communication standard has been used. This standard was originally developed for the communication between the control system and the Supervisory Control And Data Acquisition (SCADA), where an OPC UA server provides access to data through an object-oriented information model and the clients interact via standardized services, whereby each service defines a request and a response [9].

Furthermore, the usage of OPC UA allows it to easily integrate and exchange components, making the application more flexible and suitable for Industry 4.0 [10], where currently two implementation variants exist: On one hand machines are already delivered with an OPC UA server, and on the other hand a central server is in use recording historical data. In summary it can be said, that OPC UA is a suitable communication protocol not only for the communication between control systems and SCADA, but also between control systems and mobile robots [6] [11].

For securing the OPC UA connection to the client (e.g. a mobile robot) a security model is used. The server and the client have to agree on a security mechanism, which is announced by the server via the security policies [12]. In February 2018 the OPC UA specification part 14 was released with the definition of a publish/subscribe (PubSub) communication model as an alternative to the client-server model defined in part 4 [13]. With this extension published messages are forwarded to all subscribers of a topic [9]. But according to [14] the server/client architecture of OPC UA is more applicable for the communication to the enterprise network and the monitoring systems.

Securing the communication via an Industry 4.0 protocol is often not trivial, because a proper balance between performance and security must be reached. The same difficulty must be overcome in every production network, where the process control systems have higher real time requirements than IT applications. Furthermore, the enterprise network is connected to the internet, where security is more important than performance [15].

The state-of-the-art security measures in a production network are provided by the IEC 62443 [16], the cybersecurity standard for industrial automation and control systems (IACS). It contains a set of foundational requirements, standards, technical reports and related information describing how to implement and manage security measures [17]. The basic concepts presented by the IEC 62443 are network segmentation, regulation of information flow, identity and access management, system hardening, malware protection and system monitoring. It is important to say, that the focus should be on a multi-layered concept. Individual technical measures alone are not enough in today's Industry 4.0, as is evident from regular cyber-attacks using social engineering. A holistic approach should be the target, as described in [17], covering technical measures and trainings for employees.

However, there is no generally valid solution for the use of security measures, even if many companies want to use such a solution [4]. The measures must be tailored to the specific application, where IEC62443 specifies the basic requirements and methodologies as described in the following section.

\section{Methods}

The first step towards a secure production environment is based on the IEC 62443 "Industrial communication networks - Network and system security" standard. The first part of the standard sets basic requirements for an automation system. These should be met as well as possible. However, the holistic approach is particularly important here, since nonfulfilment creates a significant vulnerability in the system. The basic requirements from the IEC 62443-1-1 [16] are:

- Access control: use monitoring of devices and/or information, protecting the system against unauthorized access

- Use control: use monitoring of devices and/or information, protecting the system against unauthorized use

- Data integrity: ensuring the integrity of data in communication channels, protection against unauthorized changes

- Data confidentiality: ensuring the confidentiality of data in communication channels, protection against tapping

- Restrict dataflow: dataflow restriction in communication channels, protection against passing to unauthorized sinks

- Timely response to event: responding immediately to security vulnerabilities, notifying proper authorities and taking corrective measures

- Resource availability: ensuring the availability of network resources, system protection against DoS-attacks [18]

The goal of the proposed implementation is to fulfil the basic requirements with the principle "security by design", which means the production network should be securely designed from the ground up. This principle includes the network layout, the OPC UA communication and the mobile robot in this particular project. Taking this approach into account, avoids having to change the basic structure of the network due to emerging threats, which in most cases is expensive. Two concepts are presented here in particular, since they relate to the entire structure of the network. The first of these is "Defense in Depth". This concept refers to detecting and protecting measures in order to prevent intrusion into a system. It consists of multiple layers of security measures in order to protect the systems requiring protection. The other one builds upon the first concept. The "Zones and Conduits" model describes the division of the network into different zones. 
Criteria for segmentation can be security requirements, location or similarity of functions. The transitions between the zones are strictly monitored and are called conduits. In order to implement these concepts correctly from the start, individual zones and their conduits in the network were defined in advance. A list with all assets was created in cooperation with TÜV TRUST IT TÜV AUSTRIA GmbH. This includes an overview of the devices, (un)used interfaces, software, accessibility and their tasks. In addition to the analysis of the assets, a separate individual checklist was created for each network segment, i.e. each zone. This way it is ensured that all security measures resulting from the risk analysis are implemented.

\section{Development of a test factory}

The concepts explained in the last section were implemented in a test factory. In this factory a mobile robot (TurtleBot3 Burger) processes work orders. These orders can be created on one of the employee PCs, which are then sent to the mobile robot via the OPC UA server. The mobile robot deletes the order after execution and can then accept another one.

\subsection{Structure of a production network}

In manufacturing scenarios, production networks consist of cooperating and complexly distributed systems, aiming for long term stable operations and security by design. In order to build up sustainable defence in terms of perimeter security while simultaneously withstanding modern information security threats, lots of remedies should be taken. Furthermore, those remedies must be conscientiously, as well as continuously re-evaluated. Counteractive security measures need to be implicated in extensive efforts protecting all seven layers, while contemporaneously keeping the production environment's efficiency.

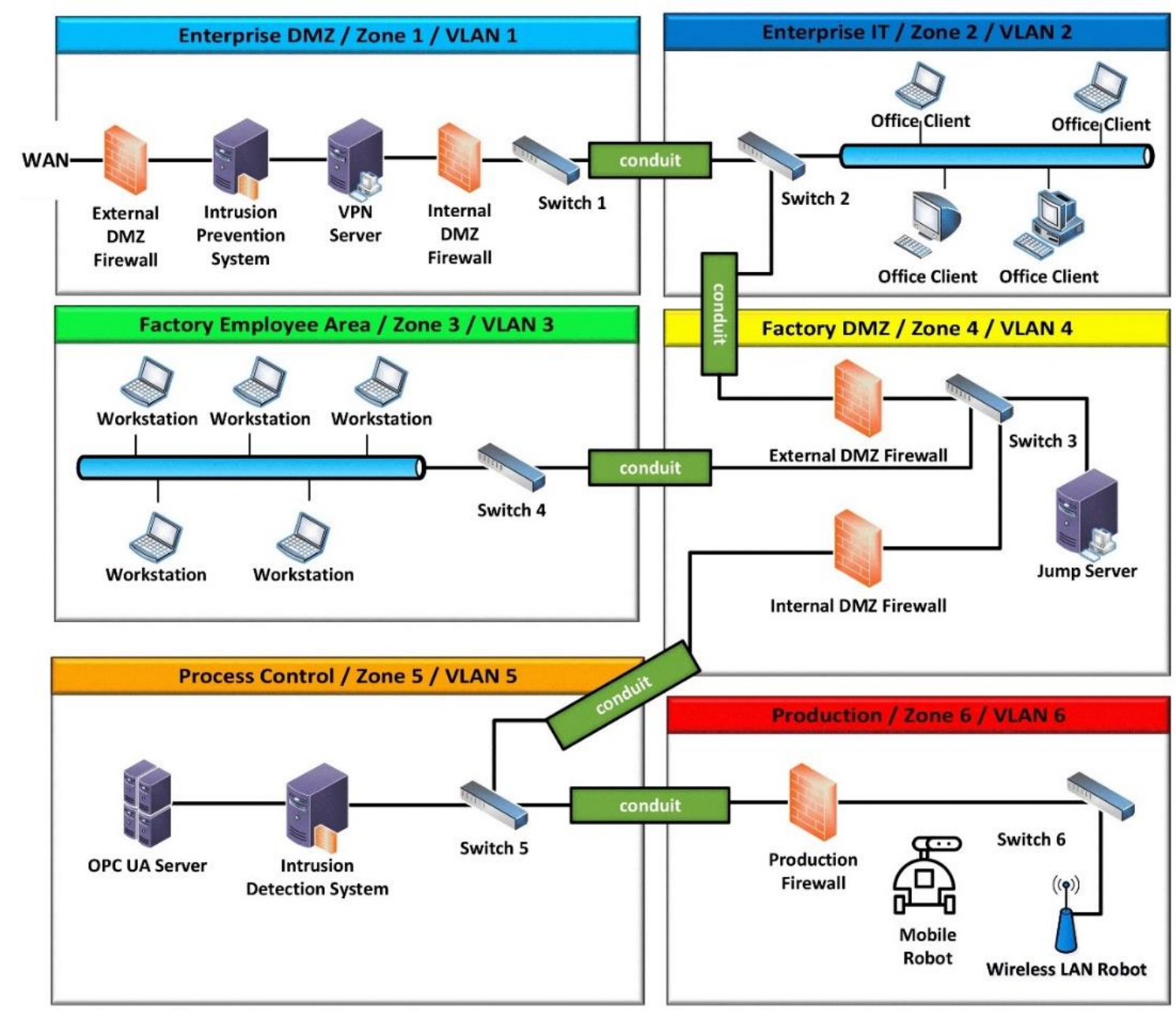

Fig. 2. Company network topology with corresponding conduits

In order to get a better insight on safety and information security needs, as well as matching the requirements from the IEC 62443-3-2 [19], it is indispensable to model (Fig. 2) and simulate the network's complexity. Considering the network's data flow transitions, it is mandatory to analyse particular information needs. 
Depending on the factory zone's or rather network's specific security requirements, defence strategies differ in relation to:

- The network zone's functional assignments

- Remote access necessity

- Network communication protocols and ports in use

- File and database access requirements

- Priority for the over whole company business

- Un-patchable legacy systems

- Continuously monitoring of production processes

- Company employee's duties and roles

In order to meet the network's data confidentiality and data integrity requirements, additional policies regarding encrypted network communication protocols such as VPN or IPsec, as well as secure application protocols SSH/TLS must be established and enforced. Connections to the production network need to be strictly controlled and monitored.

\subsection{OPC UA communication}

Further, an appropriate communication protocol has to be chosen for this factory. As said, OPC UA will be used and the two most commonly used open source libraries for setting up an OPC UA server or client are the open62541 library, used in [1][9], and the FreeOpcUa library (FreeOpcUa python and FreeOpcUa c++), used in [20][21]. According to [22], there are several open source alternatives, who are offered as a repository on GitHub. However, further development usually lies between no patches and regular patches [22].

The open62541 library is mainly developed by research institutes as the Frauenhofer IOSB. The code of the library and examples for different servers and clients can be downloaded at GitHub ${ }^{1}$. Both versions of the FreeOpcUa are developed by the community and are also available at GitHub ${ }^{2}$. All three implementations are equal in many aspects, as availability of a server and a client implementation, support of encryption and certificates and complexity. The "Medium - High" complexity is due to the fact that the server and client have to be programmed individually for each application. Compared to these libraries the UA Expert, a tool by the Unified Automation GmbH, is easy to handle due to a graphical user interface.

\begin{tabular}{|l|c|c|c|c|}
\hline \multirow{2}{*}{\multicolumn{1}{|c|}{ Criteria }} & \multicolumn{4}{c|}{ Implementation } \\
\cline { 2 - 5 } & open62541 & $\begin{array}{c}\text { FreeOpcUa } \\
\text { C++ }\end{array}$ & $\begin{array}{c}\text { FreeOpcUa } \\
\text { Python }\end{array}$ & UA Expert \\
\hline Latest release (version number) & July 2020 (1.1.2) & - & July 2020 (0.98.12) & $26.02 .2019(1.5 .1)$ \\
\hline Programming languages & C & C++ & Python & - \\
\hline Developed by & Research Institute & Community & Community & $\begin{array}{c}\text { Unified } \\
\text { Automation GmbH }\end{array}$ \\
\hline Server/Client & Server \& Client & Server \& Client & Server \& Client & Client \\
\hline Supports encryption and certificates & Yes & Yes & Yes & Yes \\
\hline Complexity & Medium - High & Medium - High & Medium - High & Low \\
\hline Certified from OPC Foundation & Yes & No & No & No \\
\hline
\end{tabular}

Table 1. Comparison of the eligible OPC UA technologies (based on: [16])

The decision which library to use was made on basis of the criterion "Certified from OPC Foundation" due to great similarities. The open62541 provides an officially certified Software Development Kit (SDK) for an OPC UA server. The sample server called "server_ctt" is conform to the "Micro Embedded Device Server" profile of the OPC Foundation and supports the security policies "Basic128Rsa15", "Basic256" and "Basic256Sha256" [23]. FreeOpcUa is not officially certified, which is why the open62541 library has been used for programming the OPC UA server and the client of the mobile robot in this case. However, UA Expert is used for the visualization of process data because it offers a graphical user interface and is easy to be operated by potential employees.

The OPC UA communication is visually presented in Fig. 3. At the PC of an employee work orders can be created with two individual programs ("Add new order" and "Delete all orders"). It is important to mention that a password is required for creation and deletion. The programs consist of a logic that validates the password and an OPC UA client transmitting the information to the process control server.

\footnotetext{
${ }^{1}$ https://github.com/open62541 [Accessed: 21.09.2020]

2 https://github.com/FreeOpcUa [Accessed: 21.09.2020]
} 


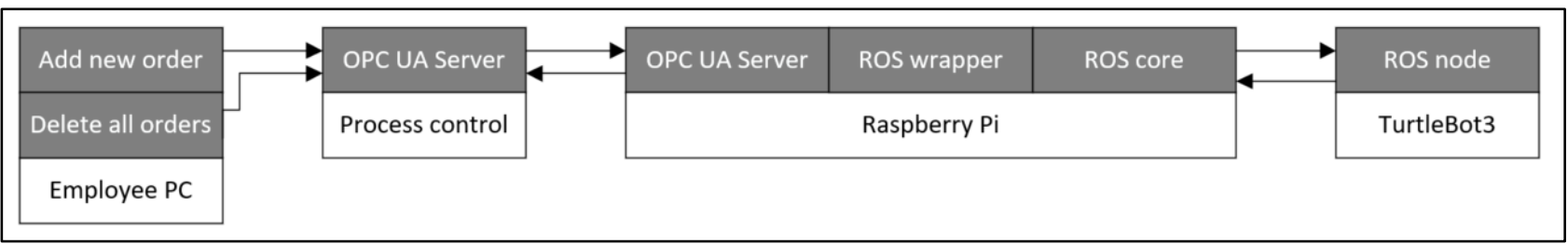

Fig. 3. Schematic representation of the factory internal communication

The OPC UA server in the process control network (VLAN5) communicates with another OPC UA server, which is located on a Raspberry Pi. The ROS core for controlling the Turtlebot 3 is also running on this Raspberry Pi. For processing the information from the server in the robot controller a ROS wrapper is necessary. This wrapper reads required information from the server and makes it available in the ROS framework. The data for the work orders are provided to the TurtleBot3 via a SSH connection. A ROS node is running on the TurtleBot3, controlling the robot (velocity and distance). Afterwards the ROS wrapper reports back to the server that the job has been processed. This first implementation has not been based on the "server_ctt" and therefore provides only a secure channel for communication, but no encryption or authentication.

\subsection{Implemented security measures}

For securing the network layer, data in between VLANs gets routed through controlled data gateways (conduits) according to IEC 62443-3-2 [24]. By having central communication gateways within the network realms, network data traffic can be monitored, and information security incidents can be reported and treated in real time. Each network zone requires different security needs depending on their security classification and impact probability:

\section{Enterprise DMZ LAN / VLAN 1}

Enterprise VLAN1 consists of two firewalls from different vendors, connects to the WAN and represents the company's external demilitarised zone. It hosts a VPN server for external employees and business partners by using a white list of public IP addresses, but also houses an intrusion prevention system to react to security incidents on the edge of the company's network. To minimize the VPN's attack surface, every VPN client must use a client certificate to authenticate itself with the service. Furthermore, cryptological keys used for IPsec and the VPN have to be at least 256 bit in case of AES and 2048 bit in case of RSA. Since the VPN server is able to connect to the WAN interface, additional security measurements have to be taken:

- Deactivating or removing of unused system applications, services, user accounts and software libraries

- Monitoring and persistent logging of VPN access control events

- Use of a host-based firewall to control traffic, network protocols and ports

- Continuously evaluating the system's security and frequently keeping the system OS up to date

- Routinely log file analysis and system backups

Enterprise IT LAN / VLAN 2

VLAN2 represents the company's office network, hosting several office work clients. VLAN2 is able to connect to the WAN and VLAN4.

\section{Factory employees / VLAN 3}

Production employee's clients are hosted in VLAN3 and are able to connect to the jump server in VLAN5. External interfaces, such as unused USB, COM or FireWire ports are deactivated. For reducing the attack surface, VLAN3 drops all connections from clients to WAN by default.

\section{Gateway / Jump server / VLAN 4}

VLAN4 hosts the internal demilitarised zone (DMZ) and is protected with an external packet filter firewall for VLAN2 and an internal packet filter firewall for VLAN5, which, for security risks, do not have the same vendor. It hosts a jump server able to connect to VLAN1, VLAN4 and VLAN5 and represents the central network access point for communicating with the OPC UA server in VLAN5.

\section{Process control / VLAN 5}

VLAN 5 is able to connect to VLAN4 and VLAN6 and hosts the OPC UA server, where TurtleBot's UPC UA client gets its tasks from. Unused interfaces were deactivated (USB, FireWire, Bluetooth,...) and the OPC UA programming libraries removed. Furthermore, it uses a host-based firewall to solely accept packets on specific ports and network protocols it is intended to use. 


\section{Production environment / VLAN 6}

This part of the network is the most delicate in terms of security. A packet filter firewall ensures it can only be accessed via the OPC UA server placed in VLAN 5. For securing the wireless connection (IEEE 802.11) between the access point (AP) and TurtleBot3 the AP uses a white list with the robot's MAC address and hides it's SSID. The packet filter firewall in place does not allow the TurtleBot 3 to connect to a WAN network, except scheduled system updates. To further reduce TurtleBot3's attack surface, a system hardening is advisable, among removing services, strong authentication and deactivating connections not necessarily used by the OS or the OPC UA client. In terms of continuous business, it is also highly advisable to frequently update and backup the robot's file system, thus reducing the downtime in case of a system failure event and reducing security risks by contemporary patching TurtleBot3's system security. In addition, further security policies are enforced and defined over all network zones:

- Users or processes have only the minimum privileges they require to perform their tasks (principle of least privilege)

- Access control monitoring regarding logins and actions, as well as network packet logging

- Default credentials are replaced on every system in the network with complex keys

- System hardening of the production environment clients by reducing / uninstalling unnecessary software applications, as well as software libraries not in use, therefore reducing the possible attack surface

- Every client connected to the production network needs to have an up to date anti-virus software installed

- Default to no discard: Only network ports needed by applications in the production environment are whitelisted, every other port is closed by default within the packet filter firewalls guarding the conduits

- Policies need to be established in consultation with thumb drives or external data within the company network

- Employees need to be aware and informed about ongoing social engineering efforts

\section{Validation of the test factory with a penetration-test}

A black box penetration test was conducted in the test factory by an expert team of TÜV TRUST IT TÜV AUSTRIA $\mathrm{GmbH}$. The defined network topology was tested for weaknesses in software and services, network security and foundational secure configurations. During a black box test, the testing person has no knowledge of the system to be tested. For comparison: A grey-box or white-box test, has partial or complete knowledge about the system.

Due to the limited time capacities for the penetration test, the test persons got access to the network of the employee control room (VLAN 3) right from the beginning and analysed the network from there. Furthermore, subnets VLAN 1 and VLAN 2 were not be implemented for the penetration test and were therefore only planned for use. The aim of the analysis was to identify security-related risks that would allow an internal attacker to compromise the security objectives. The focus in particular is on the integrity, confidentiality and availability of IT systems, processes and customer data. An overview of the results from the penetration test is shown in Table 2.

\begin{tabular}{|c|c|c|c|}
\hline Status & Strong & Potential for improvement & Problematic \\
\hline \multirow{2}{*}{ Category } & Passwords and IDs & Secure basic configuration & Network configuration \\
\cline { 2 - 4 } & & & Services and software \\
\hline
\end{tabular}

Table 2. Identified risks in the test factory by TÜV TRUST IT TÜV AUSTRIA GmbH

The lack of network segmentation is a problematic risk. The networks of the infrastructure are not separated from each other. During the audit all hosts were directly accessible from a host which was part of the DMZ network. This vulnerability must be eliminated immediately in a production network in order to prevent hosts from the DMZ from accessing the mobile robot's network and making it directly vulnerable. Furthermore, outdated versions of services and software have been used in the test factory. The vulnerabilities of the services have already been published on relevant websites, which is why they pose a particular risk. The audit also indicates the need for improvement of the base configuration. This includes unsecured network access, the missing Intrusion Detection and Intrusion Prevention System (IDS/IPS) and the insecure configuration of server services. The network access is not authenticated by MAC filters or IEEE 802.1X. In addition, the use of an IDS/IPS system is strongly recommended. The insecure configuration of server services includes the NTP server's response to mode 6 queries and the disclosure of a host using Python 2.7.17, which is already outdated. The penetration test by an expert team from TÜV TRUST IT TÜV AUSTRIA GmbH is the basis for further improvements of the test factory. The penetration test identified different weaknesses and risks, which will be fixed in order to make the use of Industry 4.0 components and their communication channels more secure.

\section{Discussion and further improvements}

Based on the contents of the IEC 62443, a secure production network could be planned and implemented, which is equipped with state-of-the-art security measures. Such a concept, planned from the very beginning, makes it possible to design a secure production network from scratch ("Security by Design"). 
Since every production plant is different, an individual concept must be used in order to achieve an optimum of security measures. Furthermore, the progressive approach of Industry 4.0 leads to the dissolution of the classical automation pyramid. However, this results in new challenges regarding security. A profound base for mastering these challenges in a production plant is the IEC 62443.

The implementation of a network based on the Digital Factory of the UAS Technikum Wien presented in this paper is suitable for industrial applications due to the implementation of the concepts of IEC 62443, the flexibility of the used communication protocol and the open source solution. The connection of real-time critical systems with IT systems, as planned for the Digital Factory, is only possible via a standardized protocol. OPC UA has proven to be the ideal communication protocol for the application in the test factory and with the open62541 library a free and open source server/client architecture could be developed, which enables a mobile robot to process work orders.

With the execution of a penetration test by an expert team from TÜV TRUST IT TÜV AUSTRIA GmbH, a number of vulnerabilities in the implementation have been revealed. For this reason, improvements will be made in the future to ensure that there are no safety incidents which could disrupt a production or, in the worst case, lead to injuries to employees by the mobile robot in use.

The next steps in order to optimize the test factory will be improvements in patch management and uninstallation of the unsupported services discovered by TÜV TRUST IT TÜV AUSTRIA GmbH. In addition, an authentication with certificates will be implemented for all clients in the network and the communication will be encrypted by TLS. Furthermore, the network segmentation will be revised and improved. From outside the network, the production plant will be only accessible via a jump server and a VPN connection. Traffic either to or from the OPC server will be constantly monitored and persistently captured by an Intrusion Prevention System (IDS). Accessing the OPA UA server and the jump server will only be possible with 2 factor authentications, using a smart card, mobile TANs or a PIN.

There will also be improvements to the OPC UA structure (Fig. 4). Only one central server will be used, which is based on the already certified server SDK and enables the authentication of the clients and the encryption of the transferred data. With the use of a central server, the Raspberry Pi, on which the ROS wrapper and the ROS core were running, will be eliminated. The client will therefore be implemented directly on the TurtleBot3.

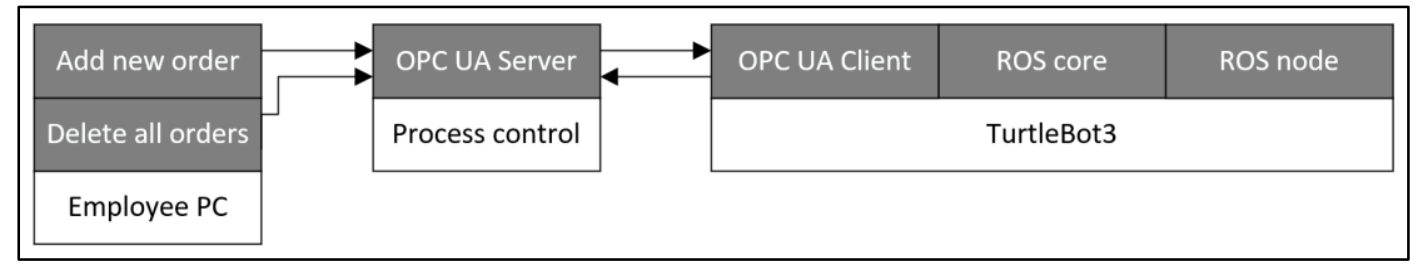

Fig. 4. Schematic representation of the improved communication

These improvements are then verified in a second penetration test in order to ensure that as many weaknesses as possible can be eliminated. However, this is not enough due to the current rapid development of Industry 4.0 technologies. In a production plant, further audits and assessments should be carried out on a regular basis in order to prevent new emerging threats. In addition, the process used for the test factory can be used as an example for the implementation in industrial applications. Based on this approach, companies are also able to build a secure production facility.

\section{Conclusion}

Currently, a big problem caused by the 4th industrial revolution is the secure combination of IT and OT in a network of cyber-physical systems. However, Austrian companies are not yet fully aware of the threats of cyber-attacks resulting from this transformation [4]. A serious problem in this context is the fact, that danger of cyber-attacks can also lead to safety risks due to the inseparable interaction.

For further analysis, different application areas of IT and OT have been combined in a test factory including a mobile robot for safety aspects. The "Defense in Depth" methodology and the "Zones and Conduits" model based on IEC 62443 form a solid basis for security in such industrial environments. An implementation of these approaches in combination with the OPC UA communication standard can increase the flexibility of the plant and have therefore been deployed. Additionally, different state-of-the-art security measures have been implemented in the individual network zones of the test factory in order to protect the mobile robot from attacks. To verify this approach, a penetration test was executed on the system by an expert team of TÜV TRUST IT TÜV AUSTRIA. The results show several vulnerabilities regarding network segmentation, outdated versions of services, the missing Intrusion Detection and Intrusion Prevention System, as well as the insecure configuration of server services.

Due to the uncovered vulnerabilities of the test factory, the security weaknesses will be corrected and further measures, such as certificates and encryption, will be added. Furthermore, the communication setup of OPC UA will be simplified. Subsequently, the test factory will be subjected to a further penetration test. In conclusion, it can be said that the development of a network in a production plant is possible on the basis of flexible Industry 4.0 technologies. 
This is especially important, since the increasing trend towards digitization and connectivity means that not only large companies can be affected by cyber-attacks. It also makes sense for small and medium-sized companies to invest in preventive measures and implement the "Defense in Depth" methodology and the "Zones and Conduits" model based on IEC 62443. Furthermore, the results of the penetration test show that it definitely makes sense to consult experts and schedule regular security audits in order to ensure that no weak points are missed.

\section{Acknowledgement}

This work was partly supported by the MA23 of the city of Vienna as a part of the project 19-05 "Sicherheit in intelligenten Produktionsumgebungen (SIP4.0)". We would like to express our special thanks to our sponsor MA23 Vienna, Austria. In this way we would also like to thank the research group of the TÜV AUSTRIA HOLDING AG and TÜV TRUST IT TÜV AUSTRIA GmbH. Without this support it would not be possible to realize this research.

\section{References}

[1] Profanter, s.; Tekat, A.; Dorofeev, K.; Rickert, M. \& Knoll, A. (2019). OPC UA versus ROS, DDS, and MQTT: Performance evaluation of Industry 4.0 protocols, Proceedings of IEEE International Conference on Industrial Technology (ICIT), February 13-15, Melbourne, Australia, ISSN 2643-2978, ISBN 978-1-5386-6376-9, pp. 955962, IEEE, Piscataway, US, DOI 10.1109/ICIT.2019.8755050

[2] Decotignie, J.-D. (2005). Ethernet-based real-time and industrial communications, Proceedings of the IEEE, Vol. 93, No. 6, pp. 1102-1117, ISSN 1558-2256

[3] Conklin, Wm. A (2016). IT vs. OT Security: A time to consider a change in CIA to include resilienc, Proceedings of 49th Hawaii International Conference on System Sciences (HICSS), January 5-8, Koloa, USA, ISSN 1530-1605, ISBN 978-0-7695-5670-3, pp. 2642-2647, IEEE, Piscataway, US, DOI 10.1109/HICSS.2016.331

[4] Federal Ministry for Climate Action, Environment, Energy, Mobility, Innovation and Technology (BMK) Austria (2020). SIGI - Sicherheit für die digitale Transformation der Produktion (Security for the digital transformation of production). Available from: https://www.bmk.gv.at/themen/innovation/publikationen/produktion/sigi.html Accessed: 2020-08-25

[5] UAS Technikum Wien (2020). Die digitale Fabrik der FH Technikum Wien (The digital factory of the UAS Technikum Wien). Available from: https://www.technikum-wien.at/digitale-fabrik/ Accessed: 2020-09-21

[6] Zhu, B.; Joseph, A. \& Sastry, S. (2011). A taxonomy of cyber attacks on SCADA systems, Proceedings of International Conference on Internet of Things and 4th International Conference on Cyber, Physical and Social Computing, October 19-22, Dalian, China, ISBN 978-1-4577-1976-9, pp. 380-388, IEEE, Piscataway, US, DOI 10.1109/iThings/CPSCom.2011.34

[7] AIT Austrian Institute of Technology GmbH (2018). Trends Und Entwicklungen In Der Österreichischen Produktion Highlights aus dem European Manufacturing Survey 2018 (Trends And Developments In Austrian Production Highlights from the European Manufacturing Survey 2018). Available from: https://www.ait.ac.at/ueber-das-ait/center/center-for-innovation-systems-policy/european-manufacturingsurvey/aktuelle-ergebnisse/ Accessed 2020-09-24

[8] Stuja, K; Poszvek, G; Wolfel, W \& Markl, E (2018). Integrated Method for the Design and Evaluation of Safety \& Secure Manufacturing Systems, Proceedings of the 29th DAAAM International Symposium, pp.0157-0163, B. Katalinic (Ed.), Published by DAAAM International, ISBN 978-3-902734-20-4, ISSN 1726-9679, Vienna, Austria, DOI: $10.2507 / 29$ th.daaam.proceedings.022

[9] Pfrommer, J.; Ebner, A.; Ravikumar, S. \& Karunakaran, B. (2018). Open source OPC UA PubSub over TSN for realtime industrial communication, Proceedings of IEEE 23rd International Conference on Emerging Technologies and Factory Automation (ETFA), September 4-7, Turin, Italy, ISSN 1946-0759, ISBN 978-1-5386-7108-5, pp. 1087-1090, IEEE, Piscataway, US, DOI 10.1109/ETFA.2018.8502479

[10] Edlinger, R.; Leimer, L.; Zauner, M. \& Froschauer, R. (2019). Towards a flexible industrial robot system architecture, In: Proceedings of the Joint ARW \& OAGM Workshop, Pichler, A.; Roth, P. M.; Sablatnig, R.; Stübl, G. \& Vincze, M. (Ed.) pp. 60-64, ISBN 978-3-85125-663-5, Steyr, Austria

[11] Pinto, T.; Arrais, R. \& Veiga, G. (2018). Bridging automation and robotics: an interprocess communication between IEC 61131-3 and ROS, Proceedings of 16th International Conference on Industrial Informatics (INDIN), July 1820, Porto, Portugal, ISSN 2378-363X, ISBN 978-1-5386-4829-2, pp. 1085-1091, IEEE, Piscataway, US, DOI 10.1109/INDIN.2018.8472057

[12] https://opcfoundation.org/developer-tools/specifications-unified-architecture/part-2-security-model/ (2018). OPC Foundation, Accessed: 2020-09-01

[13] https://opcfoundation.org/developer-tools/specifications-unified-architecture/part-14-pubsub/ (2018). OPC Foundation, Accessed: 2020-09-01

[14] Eckhardt, A.; Müller, S. \& Leurs, L. (2018). An evaluation of the applicability of OPC UA Publish Subscribe on factory automation use Cases, Proceedings of IEEE 23rd International Conference on Emerging Technologies and Factory Automation (ETFA), September 4-7, Turin, Italy, ISSN 1946-0759, ISBN 978-1-5386-7108-5, pp. 10711074, IEEE, Piscataway, US, DOI 10.1109/ETFA.2018.8502445 
[15] Cavalieri, S. \& Cutuli, G. (2010). Performance evaluation of OPC UA, Proceedings of IEEE 15th Conference on Emerging Technologies \& Factory Automation (ETFA 2010), September 13-18, Bilbao, Spain, ISSN 1946-0759, ISBN 978-1-4244-6850-8, pp. 1-8, IEEE, Piscataway, US, DOI 10.1109/ETFA.2010.5641184

[16] IEC/TS 62443-1-1:2009 (2009). Industrial communication networks - Network and system security - Part 1-1: Terminology, concepts and models

[17] Franceschett, A.L.; Souza, F P. R. A. d.; Pereira de Barros, F. L. \& De Carvalho, V.R. (2019). A holistic approach - How to achieve the state-of-art in cybersecurity for a secondary distribution automation energy system applying the IEC 62443 standard, Proceedings of IEEE PES Innovative Smart Grid Technologies Conference - Latin America (ISGT Latin America), September 15-18, Gramado, Brazil, ISSN 2643-8798, ISBN 978-1-5386-9567-8, pp. 1-5, IEEE, Piscataway, US, DOI 10.1109/ISGT-LA.2019.8895368

[18] DIN \& DKE (2017) Deutsche Normungs-Roadmap IT-Sicherheit (German Standardization Roadmap IT-Security), Available from: https://www.dke.de/de/arbeitsfelder/cybersecurity/normungs-roadmap-it-sicherheit Accessed: 2020-09-01

[19] BS IEC 62443-2-2: 2020 (2020). Security for industrial automation and control systems. Part 2-2. IACS Security Program Ratings

[20] Silveira Rocha, M.; Serpa Sestito, G.; Luis Dias, A.; Celso Turcato, A. \& Brandão, D. (2018). Performance comparison between OPC UA and MQTT for data exchange, Proceedings of Workshop on Metrology for Industry 4.0 and IoT, August 9, Brescia, Italy, ISBN 978-1-5386-2497-5, pp. 175-179, IEEE, Piscataway, US, DOI 10.1109/METROI4.2018.8428342

[21] Mizuya, T.; Okuda, M. \& Nagao, T. (2017). A case study of data acquisition from field devices using OPC UA and MQTT, Proceedings of 56th Annual Conference of the Society of Instrument and Control Engineers of Japan (SICE), November 13, Kanazawa, Japan, ISBN 978-4-907764-57-9, pp. 611-614, IEEE, Piscataway, US, DOI 10.23919/SICE.2017.8105594

[22] Haskamp, H.; Meyer, M.; Möllmann, R.; Orth, F. \&Colombo, A.W. (2017). Benchmarking of existing OPC UA implementations for Industrie 4.0-compliant digitalization solutions, Proceedings of IEEE 15th International Conference on Industrial Informatics (INDIN), November 24-26, Emden, Germany, ISSN 2378-363X, pp. 589-594, IEEE, Piscataway, US, DOI 10.1109/INDIN.2017.8104838

[23] https://open62541.org/certified-sdk (2020) open62541, Accessed: 2020-09-01

[24] IEC 62443-3-2:2020 (2020). Security for industrial automation and control systems - Part 3-2: Security risk assessment for system design 